\title{
Stability Analysis and Design of Multi-dimensional Haptic Systems
}

\author{
Gianni Bianchini* \\ Marcello Orlandesi ${ }^{\dagger}$ \\ Dipartimento di Ingegneria dell'Informazione, Università di Siena \\ Via Roma 56, 53100 Siena, Italy
}

Domenico Prattichizzo

\begin{abstract}
This paper provides a convenient framework based on passivity and optimization for stability analysis and controller design for haptic systems involving multiple devices and human operators. The proposed approach exploits peculiar features of the multi-dimensional scenario and allows for taking into account structural constraints such as operator-device configuration and controller decentralization. A method for the design and implementation of structured controllers whose impact on the realism of interaction can be qualitatively tuned, is proposed.
\end{abstract}

Index Terms: Multi-contact, Virtual coupling design, Stability, Passivity.

\section{INTRODUCTION}

Stability is a key feature in haptic interaction with virtual environments, since unwanted oscillations can impair realism and, most importantly, may be potentially harmful for the human operator. The issue of stability in this context has been addressed by several authors since the early 90's [19] and involves quite a few aspects, since the systems at hand are complex and some of their components, namely the human operators, are difficult to model. Stability has been considered from multiple viewpoints, and passivity has often been exploited in this context, since it provides a powerful tool for analyzing etherogeneous interconnected systems [12]. The fundamental paper [9] and more recent works such as [22] provide different approaches to the characterization of passivity in sampled-data systems and in particular in haptics. In $[16,15,17,18]$ a discrete-time passivity framework is proposed to deal with stability analysis and controller (virtual coupling) design also in the presence of non-passive virtual environments, and in particular [17] addresses the presence of nonlinearities. In [20], an $H_{\infty}$-type approach to the design of virtual couplings ensuring passivity and transparency is proposed. Most of the above contributions are mainly focused on the case of a single human operator interacting with a one-degree of freedom virtual environment, although also multivariable systems can be addressed to some extent.

Multi-contact interaction is an important issue in haptics [2]. Researchers from the computer haptics community, the branch of the haptic science closer to traditional robotics [21], have investigated several aspects in this scenario such as friction modeling $[14,1]$ and interaction with deformable objects [3], but mostly neglected stability issues. Recently, haptic systems where many users interact with a common virtual environment [10] and systems where a single human operator grasps virtual objects $[13,4]$ have been considered. The analysis of stability in the case of one or more human

\footnotetext{
*e-mail: giannibi@dii.unisi.it

†e-mail: orlandesi@dii.unisi.it

†e-mail: prattichizzo@dii.unisi.it
}

operators interacting with a shared virtual enviroment through multiple points of contact is a multidimensional problem that exhibits peculiar issues that need investigation.

In this paper, we deal specifically with stability analysis and controller design for haptic systems involving several human operators that interact with a common virtual environment through multiple devices. In particular, we extend some preliminary work presented in [5]. In that paper, the multi-contact scenario is addressed in a passivity-based framework which is related to the one employed in $[16,15,17,18]$, and several criteria based on linear matrix inequalities (LMI) [7] for both stability analysis and controller synthesis are proposed. Such approach suffers from a certain amount of conservatism in that it addresses multiple devices but does not distinguish between the different configurations in which one or several operators may interact with the devices themselves. As an example, the case of two haptic devices operated by a single human with two hands/fingers is considered the same as the case of two operators interacting separately with the same devices. Here, we propose a different and more general formulation of the problem that allows for reducing conservatism by introducing suitable relaxations that arise from taking explicitly into account the human-device configuration in the sense stated above. The proposed framework relies on the basic assumption that a human performing an interaction using $m$ degrees of freedom be regarded as a $m$-input, $m$-output passive operator.

As far as controller (virtual coupling) design is concerned, particular attention has to be devoted to structural constraints that may arise. Indeed, multi-contact systems may be physically distributed, and therefore the virtual coupling may share only limited information with the devices and virtual environment due to decentralization and limited communication requirements.

As a first contribution, we provide a passivity-based stability criterion that takes into account the interaction constraints outlined above. Secondly, we propose an optimization-based method that exploits such stability condition for the design of stabilizing structured controllers. In particular, we focus on a virtual coupling class that represents a virtual spring-damper network that connects the endpoints of the environment according to an a-priori assigned structure (i.e., which endpoints are connected to which endpoints) that can reflect constraints such as decentralized implementation.

The paper is organized as follows. In Section 2 we report some preliminary specific results related to passivity and to modeling and passivity-based analysis of multi-contact multi-operator haptic systems; in Section 3 we derive the sought stability condition, which is then employed in Section 4 to derive the proposed virtual coupling design method. Section 5 reports an illustrative application example and conclusions are drawn in Section 6.

\section{Notation}

For a square matrix $X, X>0(X<0)$ denotes positive (negative) definiteness, $X^{T}$ denotes transpose, and $\|X\|$ denotes some matrix norm of $X ; I_{m}$ is the $m \times m$ identity matrix. With $\mathscr{B} \mathscr{D}\left(m ; m_{1}, \ldots, m_{N}\right)$ we denote the set of $m \times m$ block-diagonal matrices whose $N$ blocks have dimensions $m_{1} \times m_{1}, \ldots, m_{N} \times m_{N}$, with 
$\sum_{i=1}^{N} m_{i}=m$. The latter notation is also used without ambiguity for block-diagonal transfer matrices of $m$-input, $m$-output linear systems and, more generally, of $m$-input, $m$-output operators. Finally, $X=\operatorname{blockdiag}\left(X_{1}, \ldots, X_{N}\right)$ denotes a block-diagonal matrix with diagonal blocks $X_{1}, \ldots, X_{N}$.

\section{Preliminaries}

\subsection{Passivity results}

The approach to stability analysis and controller design presented in this paper exploits a generalization of the passivity framework in [16]-[18], which is based upon the concepts of output strict passivity (OSP) and input strict passivity (ISP) $[12,8]$. Let us recall the definitions of OSP and ISP in both the continuous and the discretetime context.

Definition 1 (continuous-time passivity). Let $\Sigma$ be a continuoustime dynamical system with input vector $u(t) \in \mathbb{R}^{m}$, output vector $y(t) \in \mathbb{R}^{m}$, and state vector $\psi(t) \in \mathbb{R}^{n}$. If there exists a continuously differentiable positive definite function $V(\psi): \mathbb{R}^{n} \rightarrow \mathbb{R}$ (called the storage function) and $m \times m$ symmetric matrices $\Delta$ and $\Phi$ such that along all system trajectories $(\psi(t), u(t), y(t)), t \in \mathbb{R}$, the following inequality holds

$$
\dot{V}(\psi(t))<y(t)^{T} u(t)-y(t)^{T} \Delta y(t)-u(t)^{T} \Phi u(t),
$$

then, system $\Sigma$ is passive if $\Delta=\Phi=0$, output strictly passive with level $\Delta$ ( $\Delta$-OSP) if $\Delta>0, \Phi=0$, input strictly passive with level $\Phi(\Phi-$ ISP) if $\Delta=0, \Phi>0$, respectively.

Definition 2 (discrete-time passivity). Let $\Sigma_{d}$ be a discrete-time dynamical system with input vector $u(k) \in \mathbb{R}^{m}$, output vector $y(k) \in$ $\mathbb{R}^{m}$, and state vector $\psi(k) \in \mathbb{R}^{n}$. If there exists a positive definite function $V(\psi): \mathbb{R}^{n} \rightarrow \mathbb{R}$ and $m \times m$ symmetric matrices $\Delta$ and $\Phi$ such that along all system trajectories $(\psi(k), u(k), y(k)), k \in \mathbb{N}$, the following inequality holds

$$
\begin{aligned}
& \Delta V(\psi(k))=V(\psi(k+1))-V(\psi(k)) \\
& <y(k)^{T} u(k)-y(k)^{T} \Delta y(k)-u(k)^{T} \Phi u(k),
\end{aligned}
$$

then the system is passive if $\Delta=\Phi=0$, output strictly passive $(\Delta-\mathrm{OSP})$ if $\Delta>0, \Phi=0$, input strictly passive ( $\Phi-$ ISP) if $\Delta=$ $0, \Phi>0$, respectively.

Remark 1 Definitions 1 and 2, which specialize a more general concept of dissipativeness introduced in [11], differ from the commonly used notions of OSP/ISP in that the weights $\Delta$ and $\Phi$ are symmetric matrices instead of scalars. Such definitions are useful in order to address passivity problems in a MIMO context when structural constraints are present, as it will be clear in the following sections. Roughly speaking, since the passivity levels are closely related to energy dissipation, if the system at hand has a given structure, it is convenient to associate a level of dissipation to each block or component instead of the whole system. This is clearly a relaxation that is also allows for reducing conservatism when dealing with stability of interconnected systems via passivity arguments. Also, note that $\Delta$ and $\Phi$ need not necessarily be positive definite in this context: indeed, a dynamical system will be said to lack OSP (ISP) when the above definitions hold for non-positive definite $\Delta$ $(\Phi)$.

Let $\Sigma_{d}$ be a discrete-time time-invariant linear system defined by the state space representation $(A, B, C, D)$, where $A \in \mathbb{R}^{n \times n}, B \in \mathbb{R}^{n \times m}$, $C \in \mathbb{R}^{m \times n}, D \in \mathbb{R}^{m \times m}$. A straightforward extension of the standard Kalman-Yacubović-Popov lemma [8] applies.
Lemma 1 System $\Sigma_{d}$ is passive $(\Delta-O S P, \Phi-I S P)$ if and only if there exists a symmetric matrix $P \in \mathbb{R}^{n}$ such that the following two matrix inequalities hold:

$$
\left[\begin{array}{cl}
P>0 \\
A^{T} P A-P+C^{T} \Delta C & A^{T} P B-\frac{C^{T}}{2}+C^{T} \Delta D \\
B^{T} P A-\frac{C}{2}+D^{T} \Delta C & B^{T} P B-\frac{D+D^{T}}{2}+D^{T} \Delta D+\Phi
\end{array}\right]<0 .
$$

In order to address the design problems presented in this paper, we find it convenient to look for an alternative formulation of the above result in which some of the quantities involved, in particular matrices $B, D$, and $\Delta^{-1}$, appear linearly in the matrix inequalities that define the passivity condition (2). This can be accomplished via a Schur complement argument, as the following result shows.

Lemma 2 Let $\Delta>0$. System $\Sigma_{d}$ is passive $(\Delta-O S P, \Phi-I S P)$ if and only if there exist a symmetric matrix $Q \in \mathbb{R}^{n}$ and a matrix $R \in \mathbb{R}^{(n+2 m) \times n}$ satisfying the constraints

$$
\begin{aligned}
& \text { (a) } Q>0 \\
& \text { (b) }\left[\begin{array}{cc}
Q & R^{T} \\
R & S
\end{array}\right]>0 \\
& \text { (c) } \quad R=\left[\begin{array}{c}
\frac{C}{2} \\
A \\
C
\end{array}\right] Q
\end{aligned}
$$

where

$$
S=\left[\begin{array}{ccc}
\frac{D^{T}+D}{2}+\Phi & B^{T} & D^{T} \\
B & Q & 0 \\
D & 0 & \Delta^{-1}
\end{array}\right]
$$

Proof. By pre- and post- multiplication of (3)(b) by the positive definite nonsingular matrix $\left[\begin{array}{cc}Q^{-1} & 0 \\ 0 & I_{n+2 m}\end{array}\right]$ we get that (3)(b)(3)(c) are equivalent to

$$
\left[\begin{array}{cccc}
Q^{-1} & \frac{C^{T}}{2} & A^{T} & C^{T} \\
\frac{C}{2} & \frac{D+D^{T}}{2}+\Phi & B^{T} & D^{T} \\
A & B & Q & 0 \\
C & D & 0 & \Delta^{-1}
\end{array}\right]>0
$$

Taking the Schur complement with respect to the submatrix

$$
\begin{aligned}
& {\left[\begin{array}{cc}
Q & 0 \\
0 & \Delta^{-1}
\end{array}\right]>0,(4) \text { is in turn equivalent to }} \\
& \qquad\left[\begin{array}{cc}
Q^{-1} & \frac{C^{T}}{2} \\
\frac{C}{2} & \frac{D+D^{T}}{2}+\Phi
\end{array}\right] \\
& -\left[\begin{array}{cc}
A^{T} & C^{T} \\
B^{T} & D^{T}
\end{array}\right]\left[\begin{array}{cc}
Q^{-1} & 0 \\
0 & \Delta
\end{array}\right]\left[\begin{array}{ll}
A & B \\
C & D
\end{array}\right]>0
\end{aligned}
$$

which finally is equivalent to (2) once $P=Q^{-1}>0$.

\subsection{Modeling and passivity analysis of multidimen- sional haptic systems}

In this paper, we characterize multi-contact multi-operator haptic systems starting from the well-established framework of [9], [16][18]. In that framework, a haptic system is modeled as a sampleddata system (with sampling period $T$ ) resulting from the interconnection of four main components described by suitable I/O mappings (see Fig. 1): a human operator block $H$, a haptic device block $D$, a computer-simulated virtual environment $E$, and a virtual coupling $V$, whose role is to act as a controller in order to ensure the 


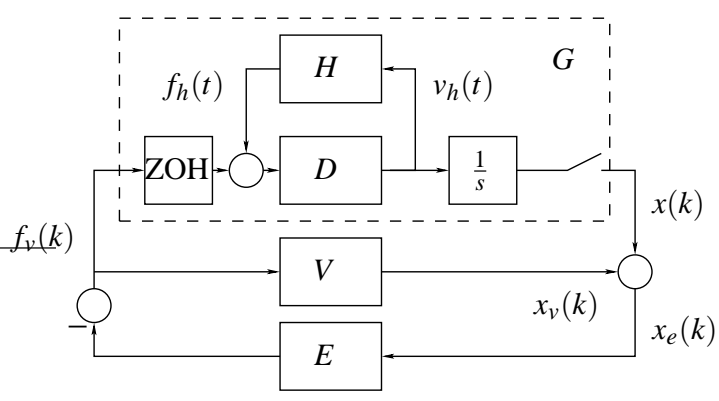

Figure 1: Haptic loop $L$

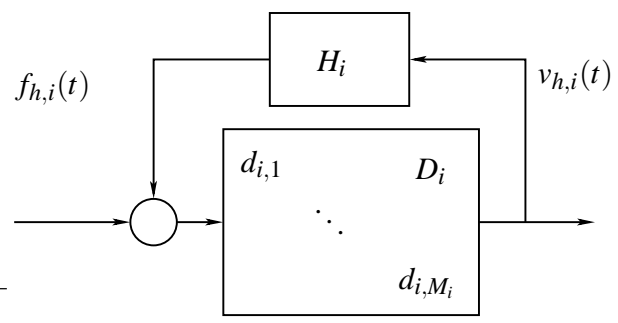

Figure 2: Interconnection of human operator $H_{i}$ with haptic devices $d_{i, 1}, \ldots, d_{i, M_{i}}$

stability of the closed-loop. The mappings $H$ and $D$ are continuoustime, while $E$ and $V$ are described by discrete-time dynamical systems. Let $L$ denote the overall loop.

In this paper, we fit the above framework to the case of $N$ human operators $H_{i}, i=1, \ldots, N$, each assumed to have $m_{i}$ degrees of freedom (DOF). Each operator $H_{i}$ is assumed to interact with $M_{i}$ different devices denoted by $d_{i, j}, j=1, \ldots, M_{i}$, where $d_{i, j}$ has $m_{i, j}$ DOF. Clearly, $m_{i}=\sum_{j=1}^{M_{i}} m_{i, j}$. All devices are coupled through a computer-simulated $m$-DOF virtual environment $E$ (with $m=\sum_{i=1}^{N} m_{i}$ ) and through a virtual coupling $V$, which are both represented by $m$-input, $m$-output discrete-time dynamical systems. In order to simplify our exposition, we assume the absence of delay in the computations and consider only the impedance causality representation of the haptic system (see [15]), although the proposed results are believed to be easily extendable to cover both the delayed case and admittance causality.

The interaction of each operator $H_{i}$ with the respective set of devices $d_{i, j}, j=1, \ldots, M_{i}$ can be described by the feedback loop in Fig. 2, in which $f_{h, i}(t) \in \mathbb{R}^{m_{i}}$ represents the generalized force vector, $v_{h, i}(t) \in \mathbb{R}^{m_{i}}$ is the generalized velocity vector presented to the operator by the devices operated by $H_{i}$, and

$$
D_{i}=\operatorname{blockdiag}\left(d_{i, 1}, \ldots, d_{i, M_{i}}\right) .
$$

It turns out that the overall system is described by the interconnection $L$ in Fig. 1, where

$$
\begin{gathered}
H=\operatorname{blockdiag}\left(H_{1}, \ldots, H_{N}\right) \in \mathscr{B} \mathscr{D}\left(m ; m_{1}, \ldots, m_{N}\right) \\
D=\operatorname{blockdiag}\left(D_{1}, \ldots, D_{N}\right) \in \mathscr{B} \mathscr{D}\left(m ; m_{1}, \ldots, m_{N}\right) \\
f_{h}(t)=\left[\begin{array}{lll}
f_{h, 1}^{T}(t) & \ldots & f_{h, N}^{T}(t)
\end{array}\right]^{T} \\
v_{h}(t)=\left[\begin{array}{llll}
v_{h, 1}^{T}(t) & \ldots & v_{h, N}^{T}(t)
\end{array}\right]^{T}
\end{gathered}
$$

and where $x(k) \in \mathbb{R}^{m}$ and $f_{v}(k) \in \mathbb{R}^{m}$ are the sampled generalized device displacement vector and sampled generalized force feedback vector, respectively.
Remark 2 Note that no peculiar structure is enforced a-priori on $V$ and $E$. However, it is often the case that the haptic device and virtual coupling are lumped together. This requirement can be easily taken into account by assuming that $V$ has a suitable block-diagonal structure as well. Clearly, additional requirements arising from decentralized computation and communication restrictions may enforce different contraints on $V$. For the sake of simplicity, in the sequel we will assume that $V$ may only be constrained to be blockdiagonal, even though the proposed approach is simply generalizable to a wide range of different structures.

Passivity-based stability analysis of haptic systems typically relies on the assumption that both the human and the device can be seen as passive operators; in particular, when an impedance causality model is employed, the device is assumed to be OSP to some extent [17]. The OSP level pertaining to a given device can be related to the amount of damping introduced into the system by the device itself. The problem of its computation has been addressed in [16] for linear devices via a robust stability argument, while in [18] it is shown, using a standard Lagrangian description of the device, that the OSP level can be related to dissipation in joint space. The latter results are easily generalizable to the OSP notion in Definition 1. Motivated by the above observations, the following assumption is made in the context of this paper.

Assumption 1 (a) Each device $d_{i, j}$ is a $\Delta_{d_{i, j}}$-OSP continuoustime dynamical system, and (b) each human block $H_{i}$ is a passive continuous-time $m_{i}$-input, $m_{i}$-output operator.

In view of Assumption 1, it is easily seen that the device block $D$ defined by (5),(6) is $\Delta_{D}$-OSP, where

$$
\Delta_{D}=\operatorname{blockdiag}\left(\Delta_{D_{1}}, \ldots, \Delta_{D_{N}}\right)
$$

being

$$
\Delta_{D_{i}}=\operatorname{blockdiag}\left(\Delta_{d_{i, 1}}, \ldots, \Delta_{d_{i, M_{i}}}\right) .
$$

In order to derive a stability criterion that takes into account the structure of the human-device block, we find it convenient to introduce a loop transformation parameterized by a diagonal matrix $\Gamma$ whose form depends on the structure itself.

Let $\gamma_{1}, \ldots, \gamma_{N}$ be strictly positive scalars, and let $\Gamma$ be the $m \times m$ diagonal matrix

$$
\Gamma=\operatorname{blockdiag}\left(\gamma_{1} I_{m_{1}}, \ldots, \gamma_{N} I_{m_{N}}\right) .
$$

The further constraint

$$
\operatorname{trace}(\Gamma)=1
$$

can be enforced without loss of generality.

For any such $\Gamma$, consider the interconnected system $L^{\Gamma}$ in Fig. 3 and denote

$$
\begin{aligned}
& f_{h}^{\Gamma}(t)=\Gamma f_{h}(t)=\left[\begin{array}{llll}
\gamma_{1} f_{h, 1}^{T}(t) & \ldots & \gamma_{N} f_{h, N}^{T}(t)
\end{array}\right]^{T} \\
& v_{h}^{\Gamma}(t)=\Gamma v_{h}(t)=\left[\begin{array}{llll}
\gamma_{1} v_{h, 1}^{T}(t) & \ldots & \gamma_{N} v_{h, N}^{T}(t)
\end{array}\right]^{T}
\end{aligned}
$$

The following result states some key properties of the loop $L^{\Gamma}$.

Theorem 1 Let Assumption 1 hold. Then, the following properties pertain to $L^{\Gamma}$ :

(P1) for any $\Gamma$ of the form (10),(11), the block $H^{\Gamma}=\Gamma H \Gamma^{-1}$ is passive,

(P2) for any $\Gamma$ of the form (10),(11), the block $D^{\Gamma}=\Gamma D \Gamma^{-1}$ is $\Delta_{D}-O S P$, where $\Delta_{D}$ is as in (8),(9),

(P3) if there exists $\Gamma$ of the form (10),(11) such that the loop $L^{\Gamma}$ is stable, i.e., the signal $v_{h}^{\Gamma}(t)$ goes to zero in steady state, then so is the original loop $L$, i.e., the velocity vector $v_{h}(t)$ goes to zero in steady state. 
there exist positive definite storage functions $V_{\hat{G}}\left(\psi_{\hat{G}}\right), V_{\hat{V}}\left(\psi_{\hat{V}}\right)$, and $V_{\hat{E}}\left(\psi_{\hat{E}}\right)$ such that (see Fig. 4)

$$
\begin{gathered}
\Delta V_{G}\left(\psi_{\hat{G}}(k)\right)<F^{T}(k) v_{G}(k)-v_{G}^{T}(k) \frac{1}{T} \Delta_{D} v_{G}(k) \\
\Delta V_{V}\left(\psi_{\hat{V}}(k)\right)<F^{T}(k) v_{V}(k)-v_{V}^{T}(k) \Delta_{\hat{V}} v_{V}(k) \\
\Delta V_{E}\left(\psi_{\hat{E}}(k)\right)<-F^{T}(k) v(k)+v^{T}(k) \Phi_{\hat{E}} v(k)
\end{gathered}
$$

Taking $V_{\hat{G}+\hat{V}}\left(\psi_{\hat{G}}, \psi_{\hat{V}}\right)=V_{\hat{G}}\left(\psi_{\hat{G}}\right)+V_{\hat{V}}\left(\psi_{\hat{V}}\right)$ we get

$$
\begin{aligned}
& \Delta V_{\hat{G}+\hat{V}}\left(\psi_{\hat{G}}(k), \psi_{\hat{V}}(k)\right)<F^{T}(k) v(k)-v^{T}(k) \Delta_{\hat{G}+\hat{V}} v(k) \\
& -\left[\begin{array}{ll}
v_{G}^{T}(k) & v_{V}^{T}(k)
\end{array}\right]\left[\begin{array}{cc}
\frac{1}{T} \Delta_{D}-\Delta_{\hat{G}+\hat{V}} & -\Delta_{\hat{G}+\hat{V}} \\
-\Delta_{\hat{G}+\hat{V}} & \Delta_{\hat{V}}-\Delta_{\hat{G}+\hat{V}}
\end{array}\right]\left[\begin{array}{c}
v_{G}(k) \\
v_{V}(k)
\end{array}\right]
\end{aligned}
$$

and hence, by the third of (16)

$$
\Delta V_{\hat{G}+\hat{V}}\left(\psi_{\hat{G}}(k), \psi_{\hat{V}}(k)\right)<F^{T}(k) v(k)-v^{T}(k) \Delta_{\hat{G}+\hat{V}} v(k)
$$

i.e., the parallel interconnection of $\hat{G}^{\Gamma}$ and $\hat{V}^{\Gamma}$ is $\Delta_{\hat{G}+\hat{V}}-$ OSP. Finally, taking $V\left(\psi_{\hat{G}}, \psi_{\hat{V}}, \psi_{\hat{E}}\right)=V_{\hat{G}+\hat{V}}\left(\psi_{\hat{G}}, \psi_{\hat{V}}\right)+V_{\hat{E}}\left(\psi_{\hat{E}}\right)$ it holds that $V\left(\psi_{\hat{G}}, \psi_{\hat{V}}, \psi_{\hat{E}}\right)$ is positive definite and, taking the fourth of (16) into account, we get

$$
\Delta V\left(\psi_{\hat{G}}(k), \psi_{\hat{V}}(k), \psi_{\hat{E}}(k)\right)<0
$$

i.e., $V\left(\psi_{\hat{G}}, \psi_{\hat{V}}, \psi_{\hat{E}}\right)$ is a Lyapunov function that proves asymptotic stability of $\hat{L}^{\Gamma}$ Therefore, the velocity vector $v_{h}(t)$ goes to zero in steady state by Theorem 3 .

\section{StRUCTURED VIRTUAL COUPLING DESIGN}

In this section, we exploit the stability condition outlined above to address the design of a virtual coupling $V$ ensuring stability of the haptic loop. To this purpose, we first provide a computationally viable criterion for calculating OSP levels $\Delta_{\hat{V}}$ that ensure stability when the virtual environment is described as a linear time-invariant system. Then, we illustrate an LMI-based procedure for the design of a linear virtual coupling $V$ of assigned structure such that the corresponding transformed virtual coupling achieves the level $\Delta_{\hat{V}}$ of OSP.

For ease of exposition, we assume the device parameters, i.e., the value of $\Delta_{D}$, to be assigned a-priori, although the proposed procedure can be modified in order to address the design of the device dissipation levels to some extent (see [6]).

\subsection{Computation of virtual coupling OSP level}

Let $\left(A_{E}, B_{E}, C_{E}, D_{E}\right)$ be a minimal state-space realization of a linear time-invariant virtual environment $E$. For given $\Gamma$, it is straightforward to check that a state space representation of the transformed virtual environment $\hat{E}^{\Gamma}$ is given by $\left(A_{\hat{E}}, B_{\hat{E}}(\Gamma), C_{\hat{E}}(\Gamma), D_{\hat{E}}(\Gamma)\right)$ where

$$
\begin{gathered}
A_{\hat{E}}=\left[\begin{array}{cc}
A_{E} & B_{E} \\
0 & I_{m}
\end{array}\right], \quad B_{\hat{E}}(\Gamma)=\left[\begin{array}{c}
B_{E} T \\
I_{m} T
\end{array}\right] \Gamma^{-1}, \\
C_{\hat{E}}(\Gamma)=\Gamma\left[\begin{array}{ll}
C_{E} & D_{E}
\end{array}\right], \quad D_{\hat{E}}(\Gamma)=\Gamma\left[D_{E} T\right] \Gamma^{-1} .
\end{gathered}
$$

In view of Theorem 4, we now show that the computation of an OSP level $\Delta_{\hat{V}}$ ensuring stability can be cast as an optimization problem involving matrix inequality constraints. In this respect, a reasonable design criterion is the minimization of some norm of $\Delta_{\hat{V}}$ subject to the stability condition of Theorem 4 .
Let us introduce the following optimization problem

$$
\begin{gathered}
\Delta_{\hat{V}}=\arg \min _{\Gamma, P, \Phi_{\hat{E}}, \Delta_{\hat{V}}, \Delta_{\hat{G}+\hat{V}}}\left\|\Delta_{\hat{V}}\right\| \\
\text { s.t. } \\
\Gamma \text { is of the form }(10),(11) \\
\Delta_{\hat{V}}>0 \\
\Delta_{\hat{G}+\hat{V}}>0 \\
P>0 \\
{\left[\begin{array}{cc}
A_{\hat{E}}^{T} P A_{\hat{E}}-P & A_{\hat{E}}^{T} P B_{\hat{E}}(\Gamma)-\frac{C_{\hat{E}}^{T}(\Gamma)}{2} \\
B_{\hat{E}}^{T}(\Gamma) P A_{\hat{E}}-\frac{C_{\hat{E}}(\Gamma)}{2} & B_{\hat{E}}^{T}(\Gamma) P B_{\hat{E}}(\Gamma)-\frac{D_{\hat{E}}(\Gamma)+D_{\hat{E}}^{T}(\Gamma)}{2}-\Phi_{\hat{E}}
\end{array}\right]<0} \\
{\left[\begin{array}{cc}
\frac{1}{T} \Delta_{D}-\Delta_{\hat{G}+\hat{V}} & -\Delta_{\hat{G}+\hat{V}} \\
-\Delta_{\hat{G}+\hat{V}} & \Delta_{\hat{V}}-\Delta_{\hat{G}+\hat{V}}
\end{array}\right]>0} \\
\Phi_{\hat{E}}-\Delta_{\hat{G}+\hat{V}}<0 .
\end{gathered}
$$

If a solution of (18) exists, then it yields a value of the level of OSP $\Delta_{\hat{V}}$ pertaining to $\hat{V}^{\Gamma}$ that satisfies the stability condition of Theorem 4 , computed so as to minimize $\left\|\Delta_{\widehat{V}}\right\|$. Note that the computation of both a suitable value of $\Gamma$ and, according to Lemma 1, a suitable ISP level $-\Phi_{\hat{E}}$ of $\hat{E}^{\Gamma}$ that are compatible with the stability condition, is implicit in the solution of the above problem.

Remark 3 By performing suitable matrix manipulations, it is possible to cast (18) into the class of Bilinear Matrix Inequality (BMI) optimization problems, and as such its solution can be computed quite efficiently by means of available solvers.

\subsection{Virtual coupling implementation}

Once $\Gamma$ and $\Delta_{\hat{V}}$ satisfing the stability condition have been obtained by means of (18), the problem becomes that of implementing a virtual coupling $V$ such that the corresponding $\hat{V}^{\Gamma}$ is $\Delta_{\hat{V}}-$ OSP, possibly taking structure constraints on $V$ into account.

In order to provide a qualitative evaluation of the impact of the virtual coupling on the the sensation felt by the users during interaction, we follow the established practice of designing the virtual coupling as a discretized spring-damper system [17, 15]. Therefore, we consider a virtual coupling $V$ of the form

$$
V=V(z)=\left[\mathbf{K}+\mathbf{B} \frac{z-1}{T z}\right]^{-1}
$$

where $\mathbf{K} \in \mathbb{R}^{m \times m}$ and $\mathbf{B} \in \mathbb{R}^{m \times m}$ will be referred to as the stiffness and damping matrix, respectively. Clearly, the transfer matrix $V(z)$ in (19) models a network of interconnected virtual springs and dampers that connect the contact endpoints to each other according to the structure of matrices $\mathbf{K}$ and $\mathbf{B}$.

It is easily seen that the level $\Delta_{\hat{V}}$ of OSP pertaining to the transformed virtual coupling $\hat{V}^{\Gamma}=\hat{V}^{\Gamma}(z)$ can be made infinite provided that $\mathbf{B}=0$ and $\mathbf{K}=\frac{2}{T} \Gamma^{-1} \Delta_{D} \Gamma$ : indeed, in this case $\hat{V}^{\Gamma}(z)$ reduces to zero (see (13) and (14)). Nevertheless, in order to achieve this, $V(z)$ is constrained to have the same structure as $\Delta_{D}$, and moreover it is not quite clear whether a virtual coupling of pure stiffness is desirable from the viewpoint of realism.

Our aim is to devise a strategy based on LMI optimization techniques, for the design of a virtual coupling of the form (19) satisfying the following requirements:

(R1) the transformed virtual coupling $\hat{V}^{\Gamma}$ is $\Delta_{\hat{V}}-\mathrm{OSP}$, where $\Delta_{\hat{V}}$ and $\Gamma$ are computed as the solution of (18), and therefore it gives rise to a stable closed loop,

(R2) $V(z)$ has an arbitrary a-priori assigned block-diagonal structure, i.e., $V(z) \in \mathscr{B} \mathscr{D}\left(m ; \bar{m}_{1}, \ldots, \bar{m}_{\bar{N}}\right)$ for given $\bar{m}_{1}, \ldots, \bar{m}_{\bar{N}}, \bar{N}$, and 
(R3) the relative amount of damping/stiffness introduced by the virtual coupling is enforced through the tuning of a free scalar parameter $\mu$.

Note that the block-diagonal structure of $V$ need not necessarily be related to the structure of $H$ and $D$, although one may find it convenient to enforce a virtual coupling structure that reflects implementation constraints such as controller decentralization (see the example section).

To proceed, rewrite $V(z)$ in (19) as

$$
V(z)=T z \bar{V}(z)
$$

where $\bar{V}(z)$ is given by

$$
\bar{V}(z)=(z I-\bar{A})^{-1} \bar{B}
$$

being

$$
\bar{A}=(\mathbf{K} T+\mathbf{B})^{-1} \mathbf{B}, \quad \bar{B}=(\mathbf{K} T+\mathbf{B})^{-1} .
$$

Conversely, we have

$$
\mathbf{K}=\frac{1}{T} \bar{B}^{-1}(I-\bar{A}), \quad \mathbf{B}=\bar{B}^{-1} \bar{A} .
$$

From (20), it turns out that the virtual coupling $V(z)$ reduces to pure stiffness in the limit case $\bar{A} \rightarrow 0$ and to pure damping for $\bar{A} \rightarrow I$. In particular, taking

$$
\bar{A}=\mu I
$$

where $0<\mu<1$, we get

$$
\mathbf{K}=\frac{1}{T}(1-\mu) \bar{B}^{-1}, \quad \mathbf{B}=\mu \bar{B}^{-1}
$$

It is then clear that the parameter $\mu$ enforces the relative amount of damping and stiffness of the virtual coupling, and that the transfer matrix $V(z)$ in (19) inherits its structure from that of $\bar{B}$, that is, if $\bar{B} \in \mathscr{B} \mathscr{D}\left(m ; \bar{m}_{1}, \ldots, \bar{m}_{\bar{N}}\right)$, then $V(z)$ has precisely the same structure.

Given $\Delta_{\hat{V}}, \Gamma$ and a scalar $0<\mu<1$, we now seek a matrix $\bar{B} \in$ $\mathscr{B} \mathscr{D}\left(m ; \bar{m}_{1}, \ldots, \bar{m}_{\bar{N}}\right)$ such that the corresponding $V(z)$ designed according to (19) and (21) satisfies the requirements (R1),(R2),(R3). In the sequel, we show that such $\bar{B}$ can be computed as the solution of an LMI feasibility problem.

The transformed virtual environment $\hat{V}^{\Gamma}$ has the transfer matrix

$$
\begin{aligned}
& \hat{V}^{\Gamma}(z)=\frac{z-1}{T z}\left[V^{\Gamma}(z)-K\right]=\frac{z-1}{T z}\left[T z \Gamma(z I-\bar{A})^{-1} \bar{B} \Gamma^{-1}-K\right] \\
& =\frac{z-1}{T z}\left[T z(z-\mu)^{-1} \Gamma \bar{B} \Gamma^{-1}-K\right] .
\end{aligned}
$$

After some manipulation, it is easily shown that $\hat{V}^{\Gamma}$ has a statespace realization $\left(A_{\hat{V}}, B_{\hat{V}}, C_{\hat{V}}, D_{\hat{V}}\right)$ of order $2 m$, where

$$
\begin{gathered}
A_{\hat{V}}=\left[\begin{array}{cc}
\mu I_{m} & 0 \\
0 & 0
\end{array}\right], B_{\hat{V}}=\left[\begin{array}{c}
\Gamma \bar{B} \Gamma^{-1} T \\
I_{m}
\end{array}\right] \\
C_{\hat{V}}=\frac{1}{T}\left[\begin{array}{ll}
(\mu-1) I_{m} & K
\end{array}\right], D_{\hat{V}}=\left[\begin{array}{c}
\Gamma \bar{B} \Gamma^{-1}-\frac{K}{T}
\end{array}\right]
\end{gathered}
$$

Note that matrices $A_{\widehat{V}}$ and $C_{\hat{V}}$ depend affinely on $\mu$, and that $B_{\widehat{V}}$ and $D_{\widehat{V}}$ depend affinely on $\bar{B}$, while both $\Gamma$ and $K=\frac{T}{2} \Delta_{D}^{-1}$ are constant at this stage.

The following result provides the sought virtual coupling design procedure.
Theorem 5 Let $\Delta_{\hat{V}}, \Delta_{D}, \Gamma$ and $0<\mu<1$ be given. Consider the following LMI feasibility problem in the unknowns $\bar{B}, R$, and $Q$ :

(a)

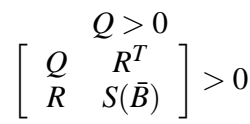

(c) $\quad R=\left[\begin{array}{c}\frac{C_{\hat{V}}}{2} \\ A_{\hat{V}} \\ C_{\hat{V}}\end{array}\right] Q$

(d) $\bar{B} \in \mathscr{B} \mathscr{D}\left(m ; \bar{m}_{1}, \ldots, \bar{m}_{\bar{N}}\right)$

where

$$
S(\bar{B})=\left[\begin{array}{ccc}
\frac{D_{\hat{V}}^{T}+D_{\hat{V}}}{2} & B_{\hat{V}}^{T} & D_{\hat{V}}^{T} \\
B_{\hat{V}} & Q & 0 \\
D_{\hat{V}} & 0 & \Delta_{\hat{V}}^{-1}
\end{array}\right]
$$

being $A_{\hat{V}}, B_{\hat{V}}, C_{\hat{V}}, D_{\hat{V}}$ as in (23).

If a solution $\bar{B}^{*}, R^{*}, Q^{*}$ of (24) exists, then the virtual coupling $V=V(z)$ designed for $\bar{B}=\bar{B}^{*}$ according to (19),(21) satisfies the requirements $(R 1),(R 2),(R 3)$.

Proof. Conditions (24)(a), (24)(b) and (24)(c) imply that $\hat{V}^{\Gamma}$ is $\Delta_{\hat{V}}$-OSP by Lemma 2 , and hence (R1) is accomplished. Moreover, $(24)(d)$ yields (R2) since $V(z)$ preserves the structure of $\bar{B}$. The fact that $\mu$ represents the relative amount of damping/stiffness (R3) is clear from (21).

\section{Application example}

We consider a haptic system in which the interaction with a 2-DOF linear virtual environment $E$ is performed through a pair of 1-DOF haptic devices $d_{x}$ and $d_{y}$. Let the two devices be characterized by OSP levels $\Delta_{d_{x}}$ and $\Delta_{d_{y}}$, respectively, according to the results in [18].

We are interested in performing stability analysis and virtual coupling design for the haptic loop in the following two cases:

i) both devices are operated by the same (2-DOF) human $H_{1}$, i.e., by a single human using both hands,

ii) the two devices are operated by two (1-DOF) humans $H_{1}$ and $\mathrm{H}_{2}$.

According to the characterization in Section 2.2, each block of the haptic loop $L$ is described by a $m$-input, $m$-output system with $m=2$. Moreover, assumptions i) and ii) enforce the following problem structures, respectively.

$$
\begin{aligned}
& \text { i) }\left\{\begin{array}{l}
N=1, m_{1}=2, M_{1}=2, d_{1,1}=d_{x}, d_{1,2}=d_{y} \\
D_{1}=\text { blockdiag }\left(d_{1,1}=d_{x}, d_{1,2}=d_{y}\right) \\
D=D_{1} \\
H=H_{1} \\
\Gamma=\left[\begin{array}{cc}
1 / 2 & 0 \\
0 & 1 / 2
\end{array}\right]
\end{array}\right. \\
& \text { ii) }\left\{\begin{array}{l}
N=2, m_{1}=m_{2}=1, M_{1}=M_{2}=1, d_{1,1}=d_{x}, d_{2,1}=d_{y} \\
D_{1}=d_{1,1}=d_{x}, D_{2}=d_{2,1}=d_{y} \\
D=\operatorname{blockdiag}\left(D_{1}, D_{2}\right) \\
H=\operatorname{blockdiag}\left(H_{1}, H_{2}\right) \\
\Gamma=\left[\begin{array}{cc}
\gamma_{1} & 0 \\
0 & \gamma_{2}
\end{array}\right], \quad \gamma_{1}, \gamma_{2}>0, \gamma_{1}+\gamma_{2}=1 .
\end{array}\right.
\end{aligned}
$$

Let the virtual environment $E$ be the backward Euler discretized version with sample period $T=0.01 \mathrm{~s}$ of the mechanical system in Fig. 5, where $x_{e}=\left[\begin{array}{ll}x_{e, 1}^{T} & x_{e_{2}}^{T}\end{array}\right]^{T}$ is the virtual environment displacement vector and $f_{v}=\left[f_{v, 1}^{T} f_{v_{2}}^{T}\right]^{T}$ is the force feedback vector 
[15] B. E. Miller. Stability of haptic systems with nonpassive virtual environments. Ph.D. Thesis, Nortwestern University, 2000.

[16] B. E. Miller, J. E. Colgate, and R. Freeman. Computational delay and free mode environment design for haptic display. In ASME Dyn. Syst. Cont. Div., 1999.

[17] B. E. Miller, J. E. Colgate, and R. Freeman. Guaranteed stability of haptic systems with nonlinear virtual environments. IEEE Transactions on Robotics and Automation, 16(6):712-719, 2000.

[18] B. E. Miller, J. E. Colgate, and R. Freeman. On the role of dissipation in haptic systems. IEEE Transactions on Robotics, 20(4):768-771, 2004.

[19] M. Minsky, M. Ouh-Young, and O. Steele. Feeling and seeing: issue in force display. ACM Trans. Comput.-Human Interactions, 24(2):235-243, 1990.

[20] P. Naghshtabrizi and J. P. Hespanha. Designing transparent stabilizing haptic controllers. In IEEE American Control Conference, Minneapolis, MI, USA, 2006.

[21] J. Salisbury, F. Conti, and F. Barbagli. Haptic rendering: introductory concepts. IEEE Computer Graphics and Animation, 24:24-32, March 2004.

[22] S. Stramigioli, C. Secchi, A. J. van der Schaft, and C. Fantuzzi. Sampled data systems passivity and discrete port-hamiltonian systems. IEEE Transactions on Robotics, 21(4):574-586, 2005. 